\title{
Coronal abundances from high-resolution X-ray data: The case of Algol
}

\author{
J. H. M. M. Schmitt and J.-U. Ness \\ Hamburger Sternwarte, Universität Hamburg, Gojenbergsweg 112, 21029 Hamburg, Germany \\ Received 7 October 2002 / Accepted 9 September 2003

\begin{abstract}
We discuss the determination of elemental abundances from high resolution X-ray data. We emphasize the need for an accurate determination of the underlying temperature structure and advocate the use of a line ratio method which allows us to utilize, first, the strongest lines observed in the X-ray spectra, and second, lines that span a rather wide temperature range. We point out the need to use continuous emission measure distributions and show via example that modeling in terms of individual temperature components yields errors of more than $50 \%$. We stress the need to derive differential emission measure distributions based on physical assumptions and considerations. We apply our methods to the Chandra LETGS spectrum of Algol and show that nitrogen is considerably enhanced compared to cosmic abundances by a factor of 2 while carbon is depleted by at least a factor of 25. Iron, silicon, and magnesium, are all depleted compared to cosmic abundances, while the noble gas neon has the relatively highest abundance.
\end{abstract}

Key words. stars: abundances - stars: activity - stars: coronae - stars: late type - X-rays: stars - stars: individual: Algol

\section{Introduction}

The determination of elemental abundances in a variety of astrophysical objects belongs to the most important tasks of observational astronomy and the understanding of the evolution of chemical elements with cosmic time is among the central themes of modern astrophysics. Elemental abundances can be measured either from absorption spectra of stellar atmospheres or from an analysis of the line emission spectrum of nebular emission. In both cases the temperature structure of the emitting object must be known before elemental abundances can be determined because for a given set of abundances plasma temperature is (often) the most important factor controlling the ionization equilibrium and hence the amount of a given type of material, say O VII or Fe XVII, in an astrophysical object.

Reliable determinations of chemical abundances are carried out from high resolution spectra. While elemental abundance determinations of stellar photospheres can also be made from a set of suitably chosen filters, abundances determined from high-resolution spectra are thought to be much more accurate and far less model-dependent. This also and specifically applies to X-ray data. The energy losses of hot thermal plasmas with temperatures above $\approx 10^{6} \mathrm{~K}$ peak in the $\mathrm{X}$-ray range and therefore the chemical composition of such plasmas, which are encountered in stellar coronae, in supernova remnants, and clusters of galaxies, can in fact only be determined from X-ray data.

Send offprint requests to: J. H. M. M. Schmitt, e-mail: jschmitt@hs.uni-hamburg.de
Continuum energy losses dominate the cooling of thermal plasmas above $\approx 10^{7} \mathrm{~K}$, while the thermal energy losses of plasma with temperatures below $\approx 10^{7} \mathrm{~K}$ are dominated by a multitude of emission lines. At those temperatures the strongest coolants are typically (albeit not exclusively) the hydrogen-and helium-like ions of the most abundant species, i.e., carbon, nitrogen, oxygen, neon, magnesium, silicon, sulfur, calcium, argon, and iron. The transitions of the hydrogen- and helium-like ions of the elements heavier than sulfur are located below $6 \AA$, and are therefore difficult to observe with high spectral resolution. Also, at temperatures below $\approx 10^{7} \mathrm{~K}$, the dominant stage of ionization for the heavier atomic species is not yet advanced to helium- or hydrogen-like ions. For example, for iron the most abundant stage of ionization at $10 \mathrm{MK}$ is boron-like Fe XXIII, at a temperature of 3 MK neon-like Fe XVII (cf. Arnaud \& Rothenflug 1985), and consequently the energy losses from iron are dominated by line emission from those ions.

The wealth of emission lines from trace elements in the $\mathrm{X}$-ray range demonstrates the potential of abundance determinations from such data. Many calculations of the total energy output of a hot collisionally ionized plasma have been made (cf. Raymond \& Smith 1977; Mewe et al. 1985; Dere et al. 1997), and the results of such calculations have been used to interpret broad-band X-ray data as available from the Einstein Observatory IPC or the ROSAT PSPC and HRI. Such plasma codes have been used to infer an energy flux from a given count rate measurement as well as to model the typically lowresolution pulse height spectra of proportional, gas scintillation 
or CCD detectors. For the spectral modeling individual spectral components are used. While the lowest resolution data can be modeled with one or two temperature components with solar abundances, higher resolution spectra require three or more temperature components with typically non-solar abundances. $\mathrm{X}$-ray detectors tend to work very efficiently at energies of $\approx 1 \mathrm{keV}$, where both the effective area of the instrument and the plasma emissivity from iron is at maximum. Deviations from solar abundances in the X-ray spectra of stars were first reported on the basis of ASCA CCD spectra of stars like Algol (cf. Antunes et al. 1994) and AR Lac (cf. White et al. 1994); also the even lower resolution PSPC spectra of some active stars were found to be better fitted with metal-depleted rather than solar-abundance plasma models (for example, Algol, cf. Ottmann \& Schmitt 1996, and CF Tuc, cf. Schmitt et al. 1996). During large flares abundance changes were inferred on the basis of spectral modeling of the time-dependent X-ray spectra. Both in AB Dor (cf. Güdel et al. 2001) as well as Algol (cf. Favata \& Schmitt 1999) the iron abundance was found to increase during the early rise phase of a flare, and then to decrease back no "normal" sub-solar abundance values.

Abundance determinations of stellar coronae based on an analysis of individual emission lines were first carried out with data from the spectrometers on board the EUVE satellite. The emission line studies based on EUVE data followed relatively closely the example of abundance determinations of the solar corona. Stern et al. (1995) and Schmitt et al. (1996) found an anomalously low iron abundance in the EUVE spectra of Algol and CF Tuc, based on an analysis of the Fe XX, Fe XXI, and Fe XXII emission lines in the XUV range and the observed continuum values. Schmitt et al. (1996) coined the term metal abundance deficiency syndrome (MADS) for this phenomenon, which is in contrast to the abundance pattern observed in the solar corona, where elements with low first ionization potential (FIP) are found to be enhanced. Drake et al. (1996) studied the presence and absence of this so-called FIP-effect in a small number of nearby stars. Using Chandra HETGS data Drake et al. (2001) study the elemental abundance of the active binary HR 1099 by means of a differential emission measure distribution computed from a Markov chain, while Brinkman et al. (2001) study the same source with the XMM-Newton reflection grating spectrometer (RGS) assigning all of the emission measure to the temperature corresponding to the peak of the line contribution function. Other abundance studies based on Chandra or XMM-Newton include Audard et al. (2001), who use a fit approach based on Chebyshev polynomials and Huenemoerder et al. (2001), who use a smoothed positive emission measure distribution function. Güdel et al. (2001) use a fitting approach based on individual temperature components to derive the elemental abundances in YY Gem using again data from the XMM-Newton RGS.

The purpose of this paper is to apply the ideas developed in solar and stellar ultraviolet emission line studies to the now available broad band and high spectral resolution X-ray data. The specific advantage of those data coming from the recent generation of X-ray spectrometers on board Chandra and XMM-Newton is that they cover the resonance lines of the hydrogen- and helium-like ions of the elements carbon, nitrogen, oxygen, neon, magnesium, and silicon. Resonance lines of the hydrogen- and helium-like ions are among the strongest observable lines and can be detected also in the weaker sources. Also, the atomic physics required for an interpretation of those lines is - probably - simpler than that required for the lines in more complex ions. We further follow quite strictly the approach that the determination of the emission measure distribution must occur in an abundance independent fashion, i.e., one either uses only lines from a given element (in practice only iron can be used) or one uses ratios of lines from the same element. The latter approach has the enormous advantage that the strongest (rather than the weaker) lines in an observed X-ray spectrum can be used for abundance determinations, once the overall continuum emission level (or possibly that of a well defined atomic species) has been fixed.

The plan of our paper is therefore as follows: we first collect the necessary formulae required for the calculation of line and continuum fluxes from an isothermal plasma at temperature $T$ with specific emphasis on the abundance dependence of these quantities. We introduce the concept of the differential emission measure (DEM); the DEM distribution is modeled by an approximation with Chebyshev polynomials on the one hand and with the help of a Gaussian distribution of magnetic loops on the other hand. The abundances computed in this fashion are juxtaposed to those computed from a more conventional analysis with individual temperature components.

\section{Abundance determination from optically thin plasma emission}

\subsection{Differential emission measure distribution}

\subsubsection{Line emission}

In this section we review the basic physics of coronal line formation in as much as relevant for elemental abundance determinations. The basic ideas of analysis have been summarized by Pottasch (1965) in a solar context. Consider the simplest case of a two-level atom in coronal equilibrium. Coronal equilibrium implies an equilibrium between collisional excitation from the lower level $l$ followed by radiative de-excitation from the excited level $u$. The emitted photon leaves the system so that in essence energy from the thermal pool has been converted into radiation. The equilibrium condition then reads as follows:

$n_{\mathrm{e} l} n_{\mathrm{ion}, l} C_{l u}=n_{\mathrm{ion}, u} A_{u l}$,

where $n_{\mathrm{e} l}, n_{\mathrm{ion}, l}$, and $n_{\mathrm{ion}, u}$ denote the number densities of electrons and ions in lower and upper state, respectively, $C_{l u}$ is the collisional rate coefficient and $A_{u l}$ the Einstein for spontaneous radiative deexcitation. The total power emitted in the transition $u l$ is given by

$P_{u l}=n_{\text {ion }, u} A_{u l} V_{\text {corona }} h v_{u l}$,

where $V_{\text {corona }}$ denotes the total (isothermal) coronal volume. Let us further assume that the considered transition is produced by a $Z$-times ionized atom from atomic species $X$; in a low density environment almost all of these ions will be in the ground 
state $l$, i.e., $n_{\text {ion }, l}=n_{X}^{Z+}$. We can therefore express $n_{\text {ion, } l}$ in the following way:

$n_{\text {ion }, l}=\frac{n_{X}^{Z+}}{n_{X}} \frac{n_{X}}{n_{\mathrm{H}}} \frac{n_{\mathrm{H}}}{n_{\mathrm{e} l}} n_{\mathrm{e}}$,

where $n_{X}$ denotes the total number density of all ions of atomic species $X$ and $n_{\mathrm{H}}$ the hydrogen/proton density. The abundance of species $X$ relative to hydrogen is denoted by $A_{X}$ and is given by

$A_{X}=\frac{n_{X}}{n_{\mathrm{H}}}$,

and in a more or less fully ionized cosmic abundance plasma we have

$n_{\mathrm{p}}=0.85 n_{\mathrm{e} l}$.

Note that for all plasmas with "reasonable" composition most of the electrons come from hydrogen and helium, so that Eq. (5) is virtually independent of the assumed elemental abundances. With these definitions the emitted power $P_{u l}$ can be written as

$P_{u l}=0.85 n_{\mathrm{el}}^{2} V_{\text {corona }} C_{l u} \frac{n_{X}^{Z+}}{n_{X}} A_{X}$.

The product $C_{l u} \frac{n_{X}^{Z+}}{n_{X}}$ depends only on temperature; we can therefore write

$P_{u l}=\Lambda_{u l}(T) E M A_{X}$,

where we have defined the volume emission measure $E M$ in the usual way through

$E M=n_{e l}^{2} V_{\text {corona }}$

and denote by $\Lambda_{u l}(T)$ the so-called line cooling function of the transition $u l$. Equation (7) illustrates the fundamental dilemma of all abundance determinations using optically thin emission in coronal equilibrium: cooling function, emission measure, and abundance all enter in the same multiplicative fashion, and therefore one has to know both emission measure and temperature in order to determine the abundance. The temperature dependence of the collisional excitation coefficients is typically of the form $C_{l u} \sim T^{-1 / 2} \mathrm{e}^{-\chi / k T}$, with $\chi$ denoting the line's excitation potential, and similar functional dependencies apply to the temperature dependence of the fractional ionization of a given ion. Consequently, a given line is sensitive over a relatively broad range of temperature with a width of typically 0.3 dex.

So far we have assumed isothermality. Stellar coronae are very likely not isothermal, and different coronal volume elements contributing to a given observed line flux in the transition $u$ to $l$ have in general different temperatures. Consider an infinitesimal volume element $\mathrm{d} V$ in a temperature range $\mathrm{d} T$, and the differential power $\mathrm{d} P_{u l}$ emitted from this volume element. The total power $P_{u l}$ can then be computed in the following way:

$$
\begin{aligned}
P_{u l}=\int \mathrm{d} P_{u l} & =A_{X} \int \mathrm{d} V n_{\mathrm{e} l}^{2} \Lambda_{u l}(T) \\
& =A_{X} \int \mathrm{d} T \xi(T) \Lambda_{u l}(T),
\end{aligned}
$$

where temperature $T$ was used to replace the integration variable $V$. The quantity $\xi(T)$ is defined through

$\xi(T)=n_{\mathrm{el}}^{2}(T) \frac{\mathrm{d} V}{\mathrm{~d} T}$

and is known as the so-called differential emission measure (DEM). Obviously we must know the differential emission measure $\xi(T)$ in order to determine the abundance $A_{X}$. Equation (9) shows, however, that the observed line flux $P_{u l}$ is an integral of the product of the line cooling function and the differential emission measure distribution. If a number of different lines with cooling functions $\Lambda_{u l}(T)$ with different temperature sensitivity are available, one obtains an integral equation with the differential emission measure distribution $\xi(T)$ as kernel. The system is a Fredholm equation of the first kind. Such integral equations are notoriously difficult to solve and, in particular, need not have unique solutions. This has been known to mathematicians for a long time, Craig and Brown (1976) were the first to point out the resulting limitations for our ability to extract physical information from spectral analysis in a cogent form.

\subsubsection{Continuum emission}

We now consider continuum radiation from a hot plasma. Continuum emission comes from (free-free) bremsstrahlung, from free-bound radiation, and two photon radiation; for temperatures $\geq 5 \mathrm{MK}$ thermal bremsstrahlung is the dominant continuum energy loss mechanism. All these continuum emission processes originate from interactions of either protons or ions with free electrons very similar to the generation of line emission. One thus expects that the dependence of the continuum emission on electron density and temperature is of the same functional form as for line emission. Indeed, Mewe et al. (1986) consider an isothermal plasma with electron number density $n_{\mathrm{e}}$ at some temperature $T$ and write the specific continuum emissivity as

$$
\frac{\mathrm{d} W}{\mathrm{~d} V \mathrm{~d} t \mathrm{~d} \lambda}=\frac{2^{5} \pi \mathrm{e}^{6}}{3 m c^{2}} \sqrt{\frac{2 \pi}{3 k m T}} n_{\mathrm{e}}^{2} \frac{1}{\lambda^{2}} \mathrm{e}^{-\frac{h c}{\lambda k T}} g_{c}(\lambda, T)
$$

here $h$ and $k$ denote Planck's and Boltzmann's constants, respectively. The quantity $g_{c}(\lambda, T)$ is the velocity-averaged Gaunt factor. It can be written as a sum of three components through

$g_{c}=g_{f f}+g_{f b}+g_{2 \gamma}$,

where $g_{f f}, g_{f b}$, and $g_{2 \gamma}$ represent the contributions from freefree, free-bound, and two photon radiation.

The different constituents of the continuum emissivity do, however, have different dependences on elemental abundances. The bremsstrahlung component comes predominantly from electron-proton collisions with most electrons due to fully ionized hydrogen and helium atoms. Under "reasonable" abundances the number of electrons from all heavier elements will be very small. In contrast, the other components do depend on trace element abundances since the free-bound radiation depends on the recombination frequency and the two-photon radiation on the number density of meta-stable He-like states, 
both of which are abundance dependent. Fortunately, for a sufficiently hot plasma electron bremsstrahlung is dominant so at least in first order the continuum energy loss is abundance independent. The specific power $\mathrm{d} P_{\lambda}$ emitted from a volume element $\mathrm{d} V$ is thus given by

$\mathrm{d} P_{\lambda}=\frac{2^{5} \pi \mathrm{e}^{6}}{3 m c^{2}} \sqrt{\frac{2 \pi}{3 k m T}} n_{\mathrm{e}}^{2} \mathrm{e}^{-\frac{h c}{\lambda k T}} \frac{1}{\lambda^{2}} g_{c}(\lambda, T) \mathrm{d} V$.

The specific power emitted from a continuous emission measure distribution described by a distribution function $\xi(T)$ is then given by

$P_{\lambda}=\frac{2^{5} \pi e^{6}}{3 m c^{2}} \int \sqrt{\frac{2 \pi}{3 k m T}} \mathrm{e}^{-\frac{h c}{\lambda k T}} \frac{1}{\lambda^{2}} g_{c}(\lambda, T) \xi(T) \mathrm{d} T$.

Thus from a mathematical point of view, the continuum emissivity is again given by an integral of the differential emission measure distribution function $\xi(T)$ with yet another kernel. In contrast to line emission, however, the continuum emission does, first, not depend sensitively on the plasma's metal abundance, and second, its temperature dependence is very different. At high temperatures, where bremsstrahlung dominates, the continuum emission is virtually independent of metal abundance since almost all electrons in a plasma come from the lightest elements. These bremsstrahlung continua are rather flat, the dominant feature being the thermal cutoff at short wavelengths, where the gratings on board Chandra and XMM-Newton have small effective areas. Therefore the continuum's thermal cutoff constrains the highest temperatures existing in a corona, but to far a lesser extent the actual run of emission measure vs. temperature.

\subsection{Constraints on and modeling of the emission measure distribution}

The temperature structure of a stellar atmosphere can be derived from the principles of radiative and hydrostatic equilibrium, the temperature structure of a magnetically confined plasma can be computed from the energy equation, if one assumes - for example - a static equilibrium. The difficulty in the latter case is, that, first, this temperature structure of an individual coronal feature is virtually independent on the form of the assumed heating, which in essence is unknown, and second, that in the stellar case one is very likely looking at the integrated emission of a large number of individual features. In other words, this integrated emission has to be described by some distribution function of the physical parameters characterizing individual coronal features, and again that distribution function is unknown. In consequence we conclude that the mathematical form of the differential emission measure distribution is a priori unknown.

Which constraints can nevertheless be imposed on the function describing the temperature structure, $\xi(T)$ ? In the following we formulate three conditions and discuss their physical and mathematical implications. Clearly, from the definition of $\xi(T)$ it follows

$\xi(T) \geq 0$
This condition looks trivial, but actually represents a rather strong constraint. Next, the DEM distributions of individual magnetic loops have (integrable) singularities at the loop top; if such DEMs are integrated over distribution functions, continuous, smooth emission measure distributions are found. We therefore assume that $\xi(T)$ is a smooth function of temperature. And finally, we know from the many low resolution X-ray data from proportional counters that the X-ray temperatures cannot extend to arbitrarily high values. We therefore assume the existence of some maximum temperature $T_{\max }$, above of which no emission measure is present, i.e.,

$\xi\left(T_{\max }\right)=0$.

In the absence of any plausible physical model we assume that the differential emission measure $\xi(T)$ can be approximated by a sum of Chebyshev polynomials; we will discuss a physical ansatz in Sect. 5.3. Of course, any other system of orthogonal polynomials or other functions could also be used. Chebyshev polynomials have unique normalization properties, and expansions of the target function into Chebyshev polynomials are useful as long as the expansion coefficients diminish rapidly with increasing order. Therefore this approach has also been taken, for example by Lemen et al. (1989) and Stern et al. (1995). Also, our available X-ray data are only sensitive to plasma hotter than some temperature $T_{\min }$, since plasma with $T<T_{\min }$ emits outside the band pass. Let us then introduce the dimensionless temperature variable $x$ defined in the closed interval $[0,1]$ through

$x=\frac{T-T_{\min }}{T_{\max }-T_{\min }}$.

Let $T_{n}(x)$ denote the Chebyshev polynomial of order $n$, satisfying the following boundary conditions $(n=0,1, \ldots)$ :

$T_{2 n}(0)=(-1)^{n} \quad T_{2 n+1}(0)=0 \quad T_{n}(1)=1$.

We seek the differential emission measure distribution $\xi(x)$ and approximate it through a sum of $M$ Chebyshev polynomials via

$\xi(x)=\sum_{i=0}^{M} a_{i} T_{i}(x)$

with coefficients $a_{i}, i=0 \ldots M$ to be determined from the data and the boundary condition

$\xi(1)=0$,

which is equivalent with Eq. (16). Since we are primarily interested in the shape of the emission measure distribution (and not in its normalization) we further impose the constraint:

$\int_{0}^{1} \mathrm{~d} x \xi(x)=1$.

Because of the properties of Chebyshev polynomials (cf. Eq. (18)) the boundary and normalization conditions Eqs. (20) and (21) translate into two conditions for the coefficients $a_{i}$, $i=0 \ldots M$ :

$\sum_{i=0}^{M} a_{i}=0$ 
and

$\sum_{i=0}^{M} a_{i} \int_{0}^{1} \mathrm{~d} x T_{i}(x)=\sum_{i=0}^{M} a_{i} I_{i}=1$,

with known coefficients $I_{i}$. Therefore the number of independent coefficients $a_{i}$ is $M-2$ and we can write the coefficients $a_{0}$ and $a_{1}$ as

$a_{1}=2 \sum_{i=2}^{M}\left(I_{i}-1\right) a_{i}-2$

and

$a_{0}=\sum_{i=2}^{M}\left(1-2 \times I_{i}\right) a_{i}-2$.

Consider now a specific spectral line denoted by some index $j$ with line cooling function $\Lambda_{j}(x)$. The measured flux $f_{j}$ of that line is given by

$$
\begin{aligned}
f_{j} & =\frac{\mathrm{e}^{-\sigma_{j} N_{\mathrm{H}}}}{4 \pi d^{2}}\left(T_{\max }-T_{\min }\right) A_{X} \int_{0}^{1} \mathrm{~d} x \Lambda_{j}(x) \xi(x) \\
& =\frac{\mathrm{e}^{-\sigma_{j} N_{\mathrm{H}}}}{4 \pi d^{2}}\left(T_{\max }-T_{\min }\right) A_{X} \sum_{i=0}^{M} a_{i} \int_{0}^{1} \mathrm{~d} x \Lambda_{j}(x) T_{i}(x),
\end{aligned}
$$

where $d$ denotes the distance to the star, $N_{\mathrm{H}}$ the hydrogen column density, and $\sigma_{j}$ the effective absorption cross section per hydrogen atom at the line's energy. With the abbreviation

$L_{j i}=\int_{0}^{1} \mathrm{~d} x \Lambda_{j}(x) T_{i}(x)$

as Chebyshev line contribution coefficients we can write

$f_{j}=\frac{\mathrm{e}^{-\sigma_{j} N_{\mathrm{H}}}}{4 \pi d^{2}}\left(T_{\max }-T_{\min }\right) A_{X} \sum_{i=0}^{M} a_{i} L_{j i}$

and one needs to determine $M+1$ coefficients $a_{i}, i=0 \ldots M$ from the data.

\subsection{Differential emission measure reconstruction from lines}

A given line flux $f_{j}$ depends both on temperature and abundance (cf. Eq. (28)), but the ratio of two emission lines from an ion of the same atomic species is clearly independent of the specific abundance of the chosen element. In order to distinguish between abundance and temperature effects on the differential emission measure distribution one should therefore work only with line ratios from the same elements (or only with lines from the same element, which in practice is feasible only for iron). If we let the index $z$ denote an emission line (or possibly a sum of emission lines of a given element) in the numerator and analogously the index $n$ the line in the denominator, we can write for the expected (abundance-independent) line ratio $\rho_{z n}$

$\rho_{z n}=\frac{f_{z}}{f_{n}}=\frac{\sum_{i=0}^{M} a_{i} L_{z i}}{\sum_{i=0}^{M} a_{i} L_{n i}}$.
Note that the idea of using line ratios is not new; for example, McIntosh (2000) has used line ratios in an attempt to determine the emission measure distribution of features in the solar corona, and Fludra \& Schmelz (1995) have used a similar approach in their analysis of X-ray flare spectra obtained with the BCS. In our context the great advantage of using line ratios lies in the fact that in this fashion the helium-like and hydrogenlike ions of many atomic species can be used. These emission lines tend to be strongest and the most easily detectable ones in any stellar corona. For the lighter elements like carbon, nitrogen, and oxygen these are in fact the only detectable lines in the X-ray range, and furthermore, atomic physics uncertainties should be smallest for such lines.

We use the double index $(z, n)$ to denote a specific line ratio and the expressions $Z_{z i}$ and $N_{n i}$ to denote the line contribution coefficients from the numerator and denominator lines entering the ratio $(z, n)$, respectively. Given a set $N$ of measured line ratios $r_{z, n}$ and errors $\sigma_{z, n}$, all of which are derived from emission lines of the same atomic species, we can determine the differential emission measure distribution by minimizing the test statistic $\chi^{2}$ defined as

$\chi^{2}=\sum_{(z, n)} \frac{\left(\frac{\sum_{i=0}^{M} a_{i} Z_{z i}}{\sum_{i=0}^{M} a_{i} N_{n i}}-r_{(z, n)}\right)^{2}}{\sigma_{(z, n)}}$

with respect to the expansion coefficients $a_{i}$ for a given set of measured line ratios and an arbitrary number $M$ of expansion coefficients. Some care is required since these coefficients are not independent; we use the boundary conditions Eqs. (24) and (25) to express the coefficients $a_{0}$ and $a_{1}$ in terms of the remaining coefficients $a_{i}, i=2 \ldots M$. We further note that multiplying any set of coefficients $a_{i}$ by some common scale factor $\Gamma$ leaves all line ratios $\rho_{(z, n)}$ invariant. As a consequence we can express the test statistic $\chi^{2}$ with the known parameters $C_{z i}$ for the numerator and $D_{n i}$ for the denominator in terms of the independent coefficients $a_{i}, i=2 \ldots M$ as

$\chi^{2}=\sum_{(z, n)} \frac{\left(\frac{\sum_{i=2}^{M} a_{i} D_{z i}}{\sum_{i=2}^{M} a_{i} C_{n i}}-r_{(z, n)}\right)^{2}}{\sigma_{(z, n)}}$.

For the latter expression derivatives can be computed analytically and minimization is straightforward. In order to ensure that the positivity constraint Eq. (15) is satisfied, we introduce a penalty function with large positive values for $\chi^{2}$ once $\xi(x)$ becomes negative at any point in the interval $x \in[0,1]$.

\subsection{Differential emission measure reconstruction from continuum}

The reconstruction of a differential emission measure distribution from a set of continuum measurements $c_{j}$ with associated errors $\sigma_{j}$ follows in analogy to the line treatment, except that obviously the appropriate continuum contribution functions must be chosen. In general the temperature sensitivity of the X-ray continuum is much less pronounced than that of individual emission lines. The most striking feature of the 
bremsstrahlung spectra is the exponential cutoff at short wavelengths resulting from the exponential decay in the number of available electrons at some given plasma temperature $T$, while at lower temperatures recombination and two photon continuum can dominate at specific wavelength bands (see discussion by Mewe et al. 1986). We also carried out test calculations of continua with "reasonable" differential emission measure distributions and found the spectral shape of these continua quite insensitive to variations in the parameters of the differential emission measure distribution. In our fits we therefore used only a small number (3) of continuum bands.

\section{Which lines to use?}

An inspection of Eq. (6) shows that the temperature dependence of the power emitted in a given spectral line is determined by two factors, i.e., first, the ionization equilibrium of the atomic species considered and second, by the electron excitation rate. The ionization equilibrium will in general be such that the fractional ionization of a given stage of ionization, say, O VII, peaks at some temperature, and at lower (higher) temperatures the predominant ionization will be lower (higher) and the emitted line flux will correspondingly change. As to the electron excitation rate, in general for excitation of a given line a certain threshold energy is required, and all electrons above this threshold value will be able to perform atomic excitations. Therefore, the excitation rates will in general increase with increasing temperature but eventually level out. Therefore, the emissivity of a given line of some species, say O VII, will peak at some temperature $T_{\max }$. Note that the line of the hydrogenlike ions are broader (with high temperature tails) than the corresponding lines from the helium-like ions, an effect caused by the ionization equilibrium. In order to illustrate this behavior we plot in Fig. 1 the line emissivity (per unit emission measure) as a function of temperature for the $\mathrm{Ly}_{\alpha}$ and He-like resonance lines (i.e., the transition ${ }^{1} \mathrm{P}_{1}-{ }^{1} \mathrm{~S}_{0}$ ) for the elements carbon, nitrogen, oxygen, neon, magnesium, and silicon according to the calculation by Mewe et al. (1985). As obvious from Fig. 1, these lines very nicely sample the temperature range between $\approx 10^{6}$ and $\approx 2 \times 10^{7} \mathrm{~K}$. These lines belong to the strongest lines of these elements in this temperature range and are easily observable in Chandra LETGS spectra.

If one now considers line ratios from the same chemical element but from two adjacent stages of ionization, say from O VII and $\mathrm{O}$ VIII, the lower ionization stage line will dominate at lower temperature and vice versa, and the line ratio will monotonically increase with temperature. In Fig. 2 we show the ratio $\rho$ of the energy flux in the $\mathrm{Ly}_{\alpha}$ line divided by the Helike $\mathrm{r}$ line as a function of temperature $T$. As is clear from Fig. 2, the line ratios $\rho$ do indeed increase monotonically with temperature. The temperatures at which $\rho$ is unity for a given atomic species increase with increasing atomic mass (and nuclear charge) reflecting the fact that more and more energy is required to establish, say, the He-like stage of ionization. $\rho$ increases by $\approx 100$ for a temperature increase of $\approx 0.5 \mathrm{dex}$, thus $\rho$ is rather temperature sensitive. Therefore a given measured line ratio can be (uniquely) converted into a temperature, however, different line ratios will in general result in different

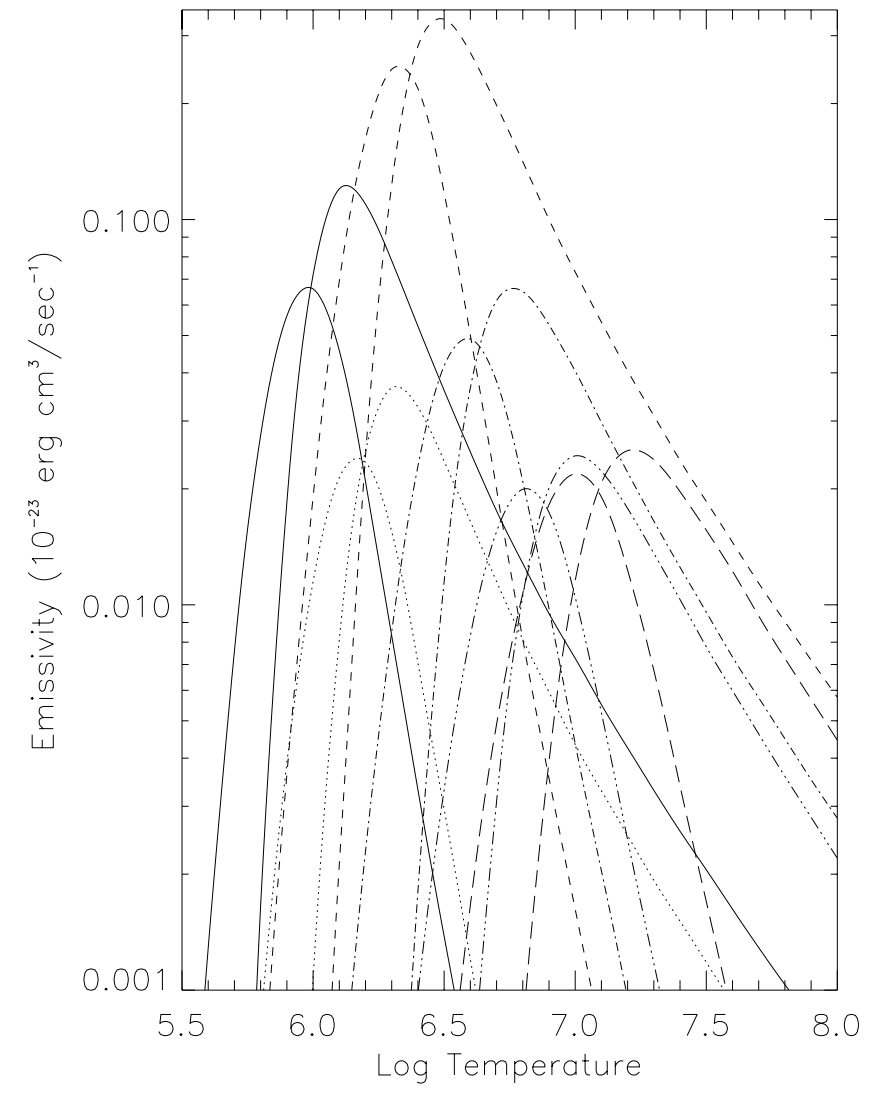

Fig. 1. Theoretical line fluxes for a cosmic abundance plasma for $\mathrm{H}-$ like and He-like resonance lines for $\mathrm{C}$ (solid lines), $\mathrm{N}$ (dotted lines), $\mathrm{O}$ (short-dashed lines), $\mathrm{Ne}$ (dash-dotted lines), $\mathrm{Mg}$ (dash-triple dotted lines), and Si (long-dashed lines) as calculated with the MEKAL code. Note, that for all elements the $\mathrm{Ly}_{\alpha}$ lines are stronger than the He-like resonance lines.

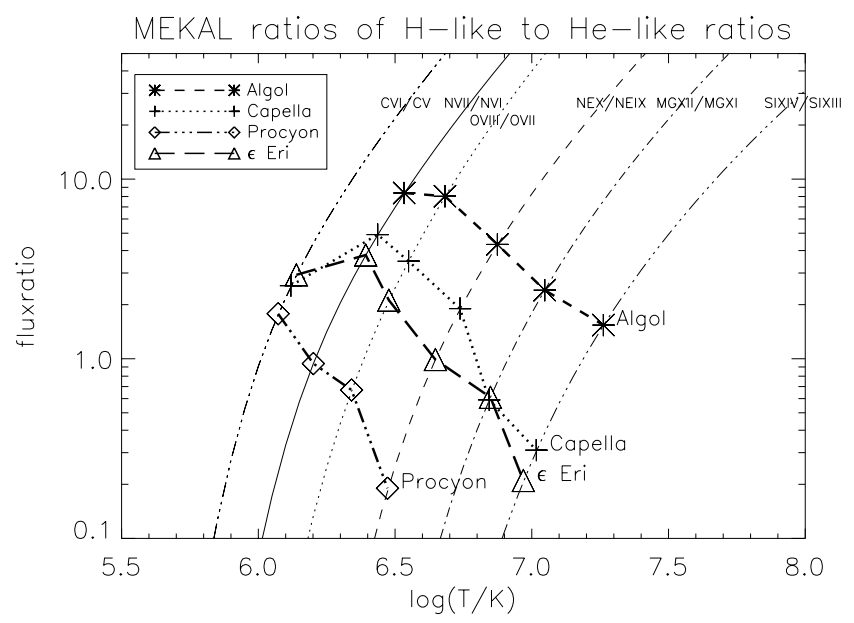

Fig. 2. Theoretical line flux ratios of $\mathrm{H}$-like by He-like resonance lines for $\mathrm{C}, \mathrm{N}, \mathrm{O}, \mathrm{Ne}, \mathrm{Mg}$, and $\mathrm{Si}$ in comparison with the measured ratios for Algol, Capella, and Procyon.

temperatures. These temperatures must not be interpreted as "isothermal" temperatures, but rather as "moment" temperatures since they depend on the differential emission measure distribution (a stellar property) and the lines' emissivity functions (an atomic property). 
How do these curves compare to observations? We included the measured data points for the stars Algol (high activity), $\epsilon$ Eri and Capella (intermediate activity), and Procyon (low activity) taken from Ness et al. (2002b), who gathered line ratios of hydrogen-like/helium-like line intensities for all ions measurable with the LETGS. As can be seen from Fig. 2, the largest value for $\rho_{\mathrm{Si}}$ is obtained for Algol $\left(\rho_{\mathrm{Si}}=1.54\right)$, while for the low-activity stars no lines from H-like or He-like silicon and magnesium are observed. For Algol the $\rho$-values increase with decreasing atomic number reaching $\rho_{\mathrm{N}}=8.37$ for nitrogen; no lines from carbon are observed. For Capella $\rho_{S i}$ and $\rho_{\mathrm{Mg}}$ are below unity, then $\rho$ increases to $\rho_{\mathrm{N}}=4.91$ for nitrogen while $\rho_{\mathrm{C}}$ is lower. For the low-activity star Procyon all $\rho$-values except for carbon $\left(\rho_{\mathrm{C}}=1.78\right)$ are below unity; the neon lines are observed only quite weakly. So clearly the available data suggest large differences in the emission measure distribution for the sample stars.

We will base our differential emission measure reconstruction solely on those lines. The disadvantage of using those lines, i.e., their formation over relatively broad temperature range, is in our opinion more than compensated by a number of advantages: first of all, the atomic physics of hydrogen- and helium-like ions is much simpler than that of more complicated ions. Second, these lines are among the strongest lines; they can therefore be measured in a large sample of stars (cf. Ness et al. 2002b) and the DEM reconstructions of different stars can be compared with each other since they are computed in the same fashion. And third, as we will show below, these lines are very likely to already contain most of the temperature information contained in stellar coronae. It is important to realize that more line ratios do not necessarily provide more information on the temperature structure; additional line ratios may either contain no or no new information or may provide conflicting information. For example, McIntosh (2000) gives the measured and calculated line ratios used in his differential emission reconstruction (cf. his Fig. 3b), which deviate by almost an order of magnitude in the worst case.

\section{Which continuum to use?}

In order to fix the overall normalization we use both the measured shape of the continuum and the absolute level of the observed continuum radiation. The first problem to be solved a problem very familiar to optical astronomers - is the correct placement of the continuum. While strong lines can be recognized easily, the sum of weak lines, each of which remains undetected, can in principle produce a "pseudo-continuum". Since specifically the LETGS covers such a large band pass it appears unlikely that over the whole instrument band pass from $5 \AA-170 \AA$ such a "pseudo-continuum" is produced, while in narrower spectral bands this may well be the case. In order to isolate the continuum we use a median filter in the following way: in a predefined wavelength region - typically we use $0.5 \AA$ - we calculate the median and use this value as the characteristic continuum level at that particular wavelength. Clearly, if too many lines are located in the wavelength bin considered, the thus derived continuum level is too high. This is specifically the case in the rather crowded region

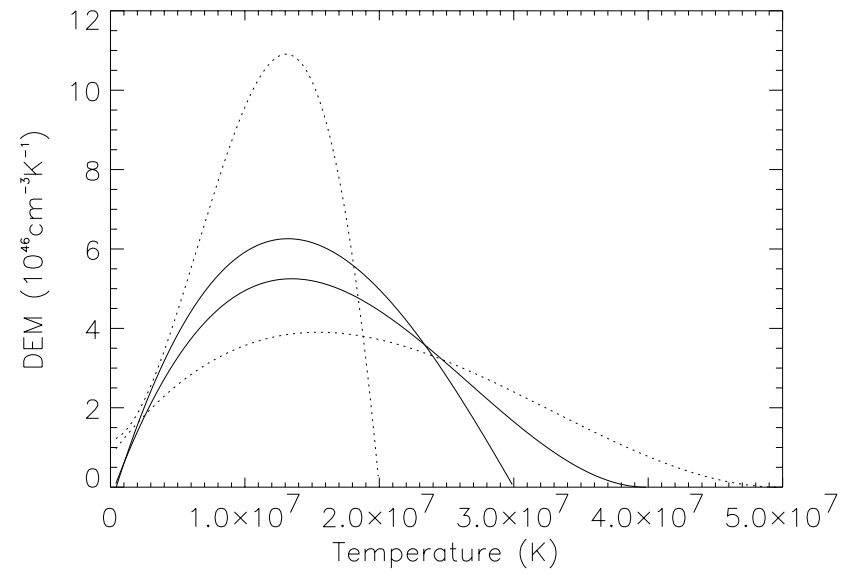

Fig. 3. Best fit differential emission measure distributions with fourthorder Chebyshev polynomials and temperatures $T_{\max }=20 \mathrm{MK}$, $30 \mathrm{MK}, 40 \mathrm{MK}$, and $50 \mathrm{MK}$; only the choices $T_{\max }=30 \mathrm{MK}$ and $T_{\max }=40 \mathrm{MK}$ yield acceptable fits to the Chandra LETGS data.

between $9 \AA$ and $18 \AA$, where it is next to impossible to reliably place any continuum. Fortunately, other spectral regions are far less crowded and do allow a rather reliable continuum placement.

\section{A worked out example: Algol}

An $80 \mathrm{ksec}$ observation of the eclipsing binary Algol has been carried out with the LETGS on board Chandra; the recorded data set and an analysis of the He-like and H-like lines has been presented by Ness et al. (2002a), while Schmitt and Ness (2002) discuss the carbon and nitrogen abundances of Algol and other giants. Here we focus on the determination of the differential emission measure distribution and elemental abundances; for a comparison of the coronal spectra of HR1099 and Algol B we refer to Drake (2003).

\subsection{DEM modeling with Chebyshev polynomials}

\subsubsection{Temperature structure}

Algol's X-ray emission is very strong and except for carbon all $\mathrm{H}$-like and $\mathrm{He}$-like lines from $\mathrm{N}, \mathrm{O}, \mathrm{Ne}, \mathrm{Mg}$, and $\mathrm{Si}$ were detected; the failure to detect carbon lines in the X-ray spectrum of Algol is model-independent and due to a nitrogen enrichment of CNO-cycle processed material (Schmitt \& Ness 2002). Thus unfortunately no information is available in the lower temperature range of the emission measure distribution from hydrogen- or helium-like lines. The flux ratio between the $\mathrm{Si} \mathrm{Ly}_{\alpha}$ and He-like resonance line exceeds unity indicating that the peak of the emission measure distribution is beyond $10 \mathrm{MK}$. We therefore consider four values of $T_{\max }$, i.e., $20 \mathrm{MK}, 30 \mathrm{MK}, 40 \mathrm{MK}$, and $50 \mathrm{MK}$. We first considered the simplest possible combination of Chebyshev polynomials with $M=4$, constrained to yielding a positive emission measure distribution. In order to prevent negative emission measures we introduced a penalty function resulting in large values whenever the reconstructed emission measure distribution has negative values in the interval between $T_{\min }$ and $T_{\max }$; we chose 
Table 1. Line ratio fit results for DEM models based on Chebyshev polynomials. The numbers given in parentheses are $\left(T_{\max }, M\right)$. For all analysed atomic species we give the observed line ratios with their errors as well as the modelled line ratios for the various models considered. The last row gives the values of the $\chi^{2}$ test statistics for the respective models.

\begin{tabular}{ccccccc}
\hline \hline Element & $\begin{array}{c}\text { Observed } \\
\text { ratio/error }\end{array}$ & $\begin{array}{c}\text { Modelled } \\
\text { line ratio } \\
(20,4)\end{array}$ & $\begin{array}{c}\text { Modelled } \\
\text { line ratio } \\
(30,4)\end{array}$ & $\begin{array}{c}\text { Modelled } \\
\text { line ratio } \\
(40,4)\end{array}$ & $\begin{array}{c}\text { Modelled } \\
\text { line ratio } \\
(50,7)\end{array}$ & $\begin{array}{c}\text { Modelled } \\
\text { line ratio } \\
(50,4)\end{array}$ \\
\hline $\mathrm{N}$ & $8.38 \pm 1.28$ & 8.85 & 9.44 & 9.21 & 9.00 & 6.66 \\
$\mathrm{O}$ & $8.00 \pm 0.71$ & 7.37 & 7.02 & 7.04 & 6.84 & 5.94 \\
$\mathrm{Ne}$ & $4.46 \pm 0.28$ & 4.80 & 4.61 & 4.76 & 4.41 & 4.77 \\
$\mathrm{Mg}$ & $2.41 \pm 0.31$ & 2.27 & 2.52 & 2.69 & 2.48 & 2.97 \\
$\mathrm{Si}$ & $1.55 \pm 0.14$ & 1.10 & 1.49 & 1.66 & 1.63 & 1.97 \\
\hline$\chi^{2}$ & & 12.45 & 3.16 & 4.82 & 3.36 & 12.89 \\
\hline
\end{tabular}

$T_{\min }=4 \times 10^{5} \mathrm{~K}$, and note that our results are not very sensitive to the specific choice of $T_{\min }$. We specifically point out that the line with the coolest peak formation temperature is the resonance line of N VI with a peak formation temperature of $\approx 10^{6.1} \mathrm{~K}$ (cf. Fig. 1) and with our Chandra LETGS data we have little information on the emission measure distribution below $10^{6} \mathrm{~K}$. For each permitted emission measure distribution the resulting line ratios of $\mathrm{N}, \mathrm{O}, \mathrm{Ne}, \mathrm{Mg}$, and $\mathrm{Si}$ were computed and compared to the observed line ratios via the $\chi^{2}$ test statistics.

In this paper we use the Chianti software package (cf. Young et al. 2003) to compute both line and continuum intensities for all modelling of Chandra LETGS data. The line intensities were computed in photon mode, for the continuum the contributions from bremsstrahlung, recombination continuum, and two photon continuum were separately computed and added. The ionization equilibrium by Mazzotta et al. (1998) was used. All calculations were carried out using cosmic abundance as quoted by Allen (1973). For the relevant elements we specifically used the values $[\mathrm{C} / \mathrm{H}]=8.52,[\mathrm{~N} / \mathrm{H}]=7.96$, $[\mathrm{O} / \mathrm{H}]=8.82,[\mathrm{Ne} / \mathrm{H}]=7.92,[\mathrm{Mg} / \mathrm{H}]=7.42,[\mathrm{Si} / \mathrm{H}]=7.52$, and $[\mathrm{Fe} / \mathrm{H}]=7.60$. Comparing these abundance values to the most recent values given by Grevesse and collaborators (1998), we find essentially identical values for $\mathrm{C}, \mathrm{N}, \mathrm{O}$ and $\mathrm{Si}$, while for the elements $\mathrm{Ne}, \mathrm{Mg}$ and $\mathrm{Fe}$ Grevesse \& Sauval (1998) give $[\mathrm{Ne} / \mathrm{H}]=8.08,[\mathrm{Mg} / \mathrm{H}]=7.58$ and $[\mathrm{Fe} / \mathrm{H}]=7.50$, i.e., values differing by factors of $1.44(\mathrm{Ne}$ and $\mathrm{Mg}$ ) and $0.79(\mathrm{Fe})$ respectively. We only give the multiplicative factors, by which our derived abundance values differ from the ones quoted by Allen (1973); in order to convert to the ones quoted by Grevesse \& Sauval (1998), the He and Mg abundances must by multiplied by 0.69 , the Fe abundance by 1.26 .

Our modeling attempts showed that already with the choice of $M=4 \operatorname{good}$ fits to the line ratios of the $\mathrm{Ly}_{\alpha}$ and He-like resonance lines can be obtained. In Fig. 3 we plot the best fit reconstructed emission measures (for the case $M=4$ ) for the peak temperatures $T_{\max }=20 \mathrm{MK}, 30 \mathrm{MK}, 40 \mathrm{MK}$, and $50 \mathrm{MK}$; both the curves for $T_{\max }=30 \mathrm{MK}$ and $40 \mathrm{MK}$ yield acceptable fits (solid lines), while the choices of $T_{\max }=20 \mathrm{MK}$ and $T_{\max }=50 \mathrm{MK}$ lead to unacceptable fits (cf. Table 1). Our formal fit results are presented in Table 1, where we give for those four best fits the resulting values of $\chi^{2}$ for the line ratio fit as

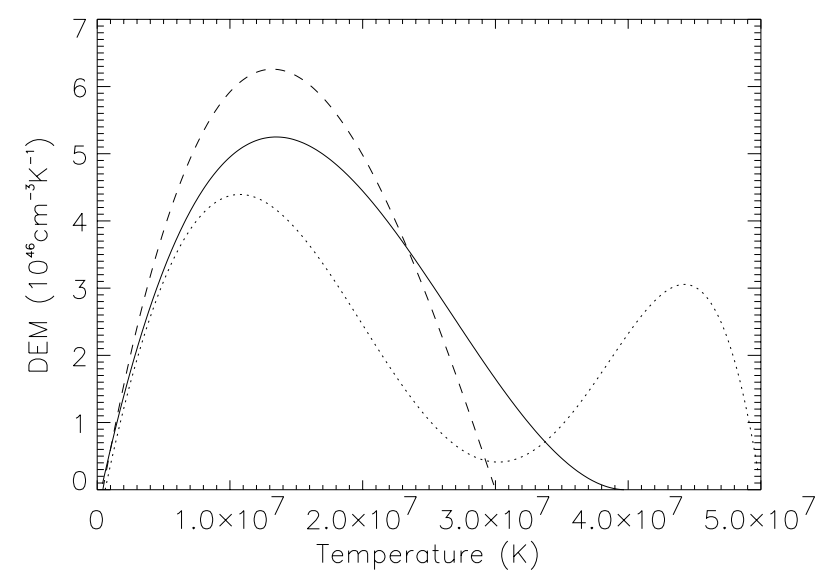

Fig. 4. Best fit differential emission measure distributions with Chebyshev polynomials with ( $T_{\max }=30 \mathrm{MK}, M=4$; dashed curve), ( $T_{\max }=40 \mathrm{MK}, M=4$; solid curve), and ( $T_{\max }=50 \mathrm{MK}, M=7$; dotted curve). All parameter choices yield acceptable fits to the Chandra LETGS line ratio data, the higher temperature yield better continuum values.

well as the modelled line ratios. A maximum temperature of $T_{\max }=20 \mathrm{MK}$ is simply too low to explain the observed ratio between $\mathrm{Ly}_{\alpha}$ and He-like resonance line for silicon. On the other hand, for the model with $T_{\max }=50 \mathrm{MK}$ and $M=4$ too much emission measure is located at high temperatures leading to an increase in $\chi^{2}$. In all fits we also checked for the goodness of fit to the continuum; in general, fits with higher temperature result in better continuum fits than lower temperatures.

Clearly, the restriction to the first four Chebyshev polynomials results in rather simple emission measure distributions, which, however, provide good line ratio fits for the correct choices of $T_{\max }$. How unique are the derived emission measure distributions? In order to assess this issue we introduced higher order Chebyshev polynomials, which result in more complicated emission measure distributions and in improved fits. In Fig. 4 we plot the best fit reconstructed emission measures for the cases $\left(T_{\max }=30 \mathrm{MK}, M=4\right),\left(T_{\max }=40 \mathrm{MK}, M=4\right)$ and $\left(T_{\max }=50 \mathrm{MK}, M=7\right)$ as a function of temperature; the resulting $\chi^{2}$ values are given in Table 1 . As can be seen from Table 1, all fits have similar goodness of fit parameters, but the resulting DEM curves are quite different. In particular, 


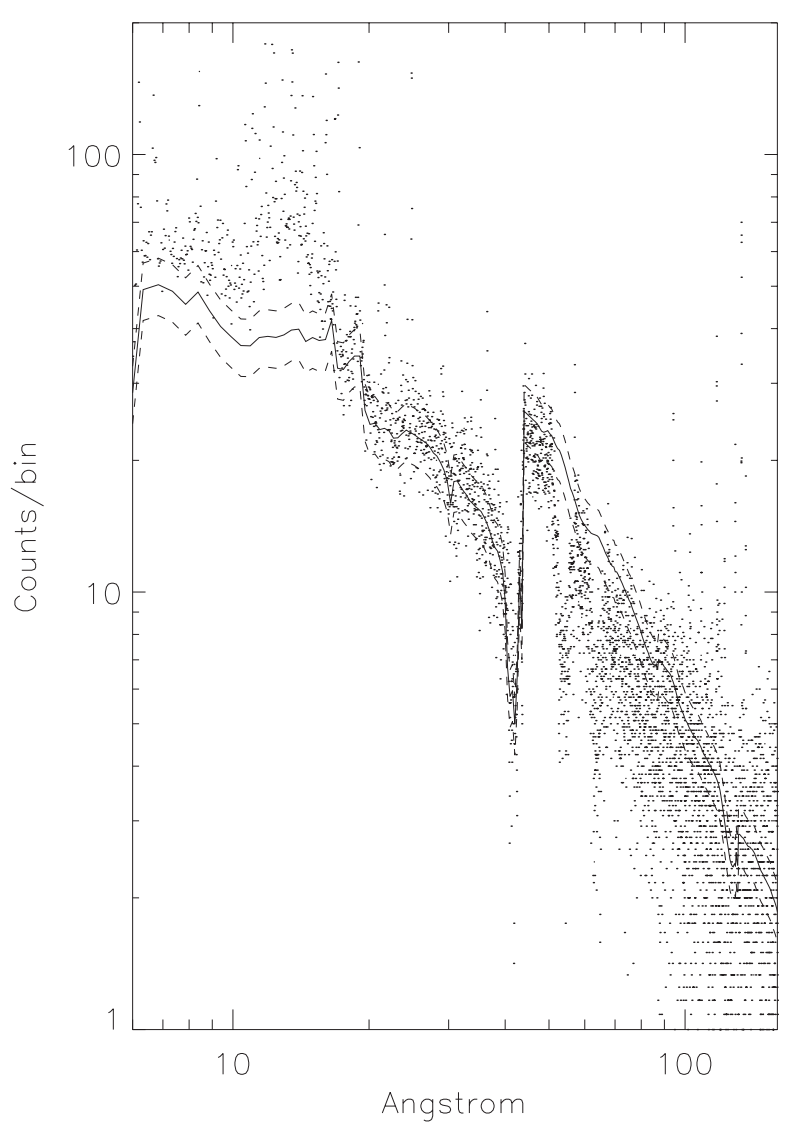

Fig. 5. Chandra LETGS spectrum for Algol binned in wavelength bins with a width of TBD $\AA$ (dots) and best fit differential emission measure model continuum for $M=4$ and $T_{\max }=40 \mathrm{MK}$ (solid line); dashed curves indicate a $15 \%$ systematic uncertainty in effective instrument areas. Note that the continuum has been calculated from the abundances actually derived for Algol.

assuming $T_{\max }=50 \mathrm{MK}$, leads to a bimodal emission measure distribution with a second peak at $45 \mathrm{MK}$, corresponding to a cutoff energy of about $3 \mathrm{keV}$. The presence of such a peak in the emission measure distribution can be readily recognized from the high energy continuum emitted by hot plasma. However, the LETGS is not particularly sensitive in that wavelength range. For XMM-Newton data, for example, simultaneously taken CCD spectra at higher energies would yield important constraints at the high temperature end of the DEM distribution which are not provided by the LETGS. From a statistical point of view the improvements in fit quality are so small that they do not warrant the introduction of additional degrees of freedom.

In order to demonstrate the overall goodness of fit of our continuum models we plot in Fig. 5 the observed Chandra LETGS for Algol (with a resolution of $0.03 \AA$; small dots) and the best fit continuum model (solid line) for the case $T_{\max }=40 \mathrm{MK}$ and $M=4$. We emphasize that in fixing the normalization we attempted only modeling the continuum, but not the individual lines; the continuum modeling includes higher orders up to order ten. The continuum was computed with the specific set of abundances calculated for Algol, but no iterative (re-)determination of abundances was performed, since the changes in the continuum level are of the order of a few percent at most. The continuum was fitted in the range $19 \AA-40 \AA$ and $70 \AA-105 \AA$. Figure 5 shows that the continuum is well fitted at short wavelengths and in the wavelength range 80-100 $\AA$. The fit is extremely poor in the wavelength range between 8 $18 \AA$; this is hardly surprising since numerous emission lines are located (cf. Fig. 1 in Ness et al. 2002a) in that wavelength region. Our fit describes quite well the carbon edge at $44 \AA$. Our fits behave badly near $\approx 53 \AA$ and $63 \AA$, where the LETGS spectra show two dips, which are instrumental and caused by gaps in the HRC-S microchannel plates. At long wavelengths our continuum fit describes the observed data, but is somewhat high. We checked our analysis procedures on the public Chandra LETGS data (500 ksec) of the isolated neutron star RXJ1856.5-3754 and found very good agreement between our results and those published in the literature. We have at present no satisfactory explanation for the discrepancy at longer wavelengths. On the one hand our choice of median filtering has some bias towards higher values because the filtering procedure essentially regards spectral lines as background fluctuations. On the other hand errors in the instrument calibration can also not be ruled out; calibrations in the EUV are notoriously difficult and an error in the relative calibration between the long- and short wavelength region will also help to reduce the observed discrepancy. An even larger absorption column towards Algol would also improve the fit.

\subsubsection{Abundances}

With the reconstructed abundance independent emission measure distributions (cf. Fig. 3) and the measured line fluxes we can now calculate elemental abundances. Specifically, we can calculate for each observed spectral line that value of abundance which forces agreement between predicted and observed line fluxes. Obviously, the abundances for a given chemical element will depend on the specific lines used and any errors in the atomic parameters and temperature structure will propagate into the derived abundances; our results for various lines are summarized in Table 2. All abundance values quoted are relative to the values $[\mathrm{C} / \mathrm{H}]=8.52,[\mathrm{~N} / \mathrm{H}]=7.96$, $[\mathrm{O} / \mathrm{H}]=8.82,[\mathrm{Ne} / \mathrm{H}]=7.92,[\mathrm{Mg} / \mathrm{H}]=7.42,[\mathrm{Si} / \mathrm{H}]=7.52$, and $[\mathrm{Fe} / \mathrm{H}]=7.60$. An inspection of Table 2 shows that $\mathrm{H}$ - and $\mathrm{He}-$ like lines give consistent abundance values; this is of course no surprise, since the adopted differential emission measure distribution fits the line ratios very well. The upper limit derived from the absence of the $\mathrm{C}$ VI $\mathrm{Ly}_{\alpha}$ line implies that the carbon abundance (relative to solar) is at the level of less than 10 percent, i.e., most of the carbon has been depleted. No iron lines have been used for the DEM reconstruction. Inspection of Table 2 shows that the nitrogen abundances for accpetable models lie between 1.9-2.9, the oxygen abundances between $0.25-0.36$, the neon abundances between $0.95-1.34$, the magnesium abundances between $0.48-0.68$, and those of silicon between $0.42-0.61$. The Fe abundances (for the best fit DEM models) vary from $0.10-0.49$ depending on the lines chosen. These discrepancies are of course worrisome, but they are small compared to the discrepancies found in solar work 
Table 2. Abundance determinations for DEM models based on Chebyshev polynomials; listed are the elements and lines used (first two columns), the measured number of counts, the modelled number of counts as well as the abundance relative to the Allen (1973) abundance values for the various models characterized by number of polynomials and peak temperature. Last row indicates whether fits are acceptable "acc" or unacceptable "not acc".

\begin{tabular}{|l|rr|rr|rr|rr|rr|rr|}
\hline \hline Ion & $\begin{array}{r}\text { Line } \\
(\AA)\end{array}$ & Obs. & Mod. & $(7,50)$ & Mod. & $(4,30)$ & Mod. & $(4,40)$ & Mod. & (4, 50) & Mod. & $(4,20)$ \\
& & & & & & & & & & \\
\hline C VI & 33.74 & $<60$ & 588.2 & $<0.10$ & 763.1 & $<0.08$ & 660.5 & $<0.09$ & 659.8 & $<0.09$ & 908.1 & $<0.05$ \\
N VI & 24.785 & 1119.1 & 416.9 & 2.68 & 567.4 & 1.97 & 501.1 & 2.23 & 467.7 & 2.39 & 673.0 & 1.71 \\
N VII & 28.79 & 141.3 & 48.6 & 2.91 & 63.3 & 2.23 & 57.0 & 2.48 & 54.4 & 2.59 & 76.9 & 1.84 \\
O VIII & 18.97 & 2883.0 & 7925.2 & 0.36 & 10289.0 & 0.28 & 9057.4 & 0.32 & 8890.1 & 0.32 & 11749.0 & 0.25 \\
O VII & 21.6 & 262.5 & 836.7 & 0.31 & 1059.7 & 0.25 & 930.1 & 0.28 & 938.5 & 0.28 & 1251.5 & 0.21 \\
Ne X & 12.138 & 2481.5 & 1956.4 & 1.27 & 2602.2 & 0.95 & 2300.1 & 1.09 & 2194.7 & 1.13 & 2996.8 & 0.83 \\
Ne IX & 13.45 & 665.0 & 497.8 & 1.34 & 633.5 & 1.05 & 542.4 & 1.23 & 558.4 & 1.19 & 710.7 & 0.94 \\
Mg XII & 8.43 & 578.0 & 878.0 & 0.66 & 1196.3 & 0.48 & 1086.1 & 0.53 & 985.0 & 0.59 & 1406.1 & 0.41 \\
Mg XI & 9.17 & 224.1 & 329.3 & 0.68 & 439.7 & 0.51 & 374.9 & 0.60 & 369.4 & 0.61 & 514.6 & 0.44 \\
Si XIV & 6.15 & 658.3 & 1135.8 & 0.58 & 1504.8 & 0.44 & 1452.2 & 0.45 & 1274.1 & 0.52 & 1784.5 & 0.37 \\
Si XIII & 6.65 & 480.7 & 784.7 & 0.61 & 1132.1 & 0.42 & 983.0 & 0.49 & 880.3 & 0.55 & 1618.6 & 0.41 \\
Fe XVII & 15.01 & 1018.4 & 5322.6 & 0.19 & 6740.0 & 0.15 & 5714.8 & 0.18 & 5970.6 & 0.17 & 7286.7 & 0.14 \\
Fe XVII & 15.27 & 364.7 & 1286.1 & 0.28 & 1628.1 & 0.22 & 1380.6 & 0.26 & 1442.6 & 0.25 & 1760.1 & 0.21 \\
Fe XVII & 17.07 & 1124.5 & 2278.8 & 0.49 & 2879.7 & 0.39 & 2443.5 & 0.46 & 2556.2 & 0.44 & 3109.9 & 0.36 \\
Fe XVII & 93.92 & 258.0 & 2023.9 & 0.13 & 2599.7 & 0.10 & 2196.2 & 0.12 & 2270.3 & 0.11 & 2857.9 & 0.09 \\
Fe XIX & 108.37 & 203.5 & 1193.6 & 0.17 & 1571.0 & 0.13 & 1323.1 & 0.15 & 1339.0 & 0.15 & 1817.1 & 0.11 \\
Fe XIX & 101.55 & 180.9 & 444.9 & 0.41 & 585.5 & 0.31 & 493.2 & 0.37 & 499.0 & 0.36 & 677.3 & 0.25 \\
Fe XXI & 128.73 & 266.32 & 1914.5 & 0.14 & 2725.2 & 0.10 & 2300.5 & 0.12 & 2147.8 & 0.12 & 3730.5 & 0.07 \\
Fe XXIII & 132.85 & 1178.6 & 5561.9 & 0.21 & 8505.0 & 0.14 & 7508.5 & 0.16 & 6250.8 & 0.19 & 11021.3 & 0.11 \\
\hline & & & & acc & & acc & & acc & & not acc & not acc \\
\hline
\end{tabular}

(cf. McIntosh 2000). Also, some Fe lines consistently yield abundance values inconsistent with those derived from other lines. For example, the Fe XVII 17.07 $\AA$ line yields larger values than the neighboring $15 \AA$ line, and the Fe XIX $101.55 \AA$ line gives much higher values than the other Fe XUV lines, indicating systematic problems with those lines. Table 2 also reveals that the Fe abundances derived from XUV lines (with the exception of Fe XIX 101.55 $\AA$ ) are in general lower than those of X-ray lines. Whether this is due to atomic physics or instrumental problems is difficult to say at present. The errors in the effective area calibration of the LETGS are thought to be of the order $\pm 15 \%$ at most; the magnitude of this effect appears to be too small to account for the observed discrepancies. Another issue is the value of the interstellar absorption column density; for our analysis we used the value of $5 \times 10^{-18} \mathrm{~cm}^{-3}$, and higher values would reduce, smaller values increase the discrepancy between model and observations.

\subsection{Temperature component analysis}

\subsubsection{Temperature structure}

It is of course also possible to carry out the analysis of the line ratio data in a more traditional way using individual, discrete temperature components. In this approach one uses $L$ independent spectral components with temperatures $T_{l}, l=1 \ldots L$ and emission measures $A_{l}$. One clearly ought to demand

$A_{l} \geq 0$ and, since we are using line ratios, any solution is only determined up to a multiplicative factor which needs to be fixed by the continuum. We use the constraint

$\sum_{l=1}^{L} A_{l}=1$

and therefore a solution with $L$ spectral components has $2 L-1$ fit parameters. In Table 3 we present the results of our analysis of the available line-ratios for Algol using individual temperature components; we list the best fit temperatures and amplitudes as well as the goodness of fit parameter $\chi^{2}$. An inspection of Table 3 shows that a good fit to the observed line ratios measured for the $\mathrm{Ly}_{\alpha^{-}}$and He-like resonance lines for $\mathrm{N}, \mathrm{O}, \mathrm{Ne}$, $\mathrm{Mg}$, and Si requires four spectral components with a total of seven adjustable parameters. Descriptions with fewer components and fewer adjustable parameters cannot explain the observed line ratios; the test statistic $\chi^{2}$ depends - not surprisingly - sensitively on the number of spectral components, but three components, yielding a test statistic $\chi_{\text {min }}^{2}=17.15$, are just not sufficient, while the introduction of a fourth and fifth component reduces the level of $\chi^{2}$ to below unity. The necessity to introduce 7 parameters in order to fit 5 data points is a nightmare for every statistician and one wonders about the effective number of degrees of freedom. Presumably these problems have to do with the positivity constraint Eq. (32), which severely limits the available solution space. Unfortunately, the resulting best fit temperature quadruple is not unique. If one accepts a limit of $\chi_{\lim }^{2} \leq 6$ as a threshold below which solutions are accepted uncertainties of up to 0.2 dex in $\log T$ result. 
Table 3. Abundance determinations for discrete temperature component models; listed are the elements and lines used (first two columns), the model parameters in parentheses indicating number of temperature components and value of $\chi^{2}$ as well as the individually derived abundances relative to cosmic abundances. Last row indicates whether model gives acceptable "acc" or unacceptable fits "not acc".

\begin{tabular}{lrrrr}
\hline \hline Ion & Line $(\AA)$ & $(3,9.1)$ & $(4,2.26)$ & $(5,0.83)$ \\
\hline C VI & 33.74 & $<0.06$ & $<0.06$ & $<0.06$ \\
N VI & 28.79 & 1.54 & 1.41 & 1.45 \\
N VII & 24.785 & 1.35 & 1.41 & 1.45 \\
O VII & 21.6 & 0.19 & 0.17 & 0.19 \\
O VIII & 18.97 & 0.20 & 0.19 & 0.19 \\
Ne IX & 13.45 & 0.68 & 0.77 & 0.74 \\
Ne X & 12.138 & 0.58 & 0.69 & 0.69 \\
Mg XI & 9.17 & 0.28 & 0.33 & 0.33 \\
Mg XII & 8.43 & 0.39 & 0.36 & 0.37 \\
Si XIII & 6.65 & 0.33 & 0.35 & 0.36 \\
Si XIV & 6.15 & 0.34 & 0.34 & 0.34 \\
Fe XVII & 15.01 & 0.06 & 0.09 & 0.09 \\
Fe XVII & 15.27 & 0.09 & 0.13 & 0.13 \\
Fe XVII & 17.07 & 0.16 & 0.24 & 0.23 \\
Fe XVII & 93.92 & 0.05 & 0.05 & 0.05 \\
Fe XX & 101.55 & 0.24 & 0.15 & 0.06 \\
Fe XIX & 108.37 & 0.10 & 0.06 & 0.06 \\
Fe XXI & 128.73 & 0.16 & 0.14 & 0.13 \\
Fe XXIII & 132.85 & 0.11 & 0.15 & 0.15 \\
\hline & & not acc & acc & acc \\
\hline
\end{tabular}

\subsubsection{Abundances}

One expects that the derived abundances depend sensitively on the choice of the adopted temperature components if one is working with a small number of temperature components. These expectations are verified by an inspection of Table 3, where we list the abundances derived from our temperature fits. In Table 3 we list the derived elemental abundances for each discrete temperature solution for three, four, and five individual components (list in the first row). The depletion of carbon is recognized independent of the chosen number of temperature components, while abundances for individual elements and lines can but must not vary considerably. For example, the $\mathrm{O}$ abundance derived from O VIII $18.97 \AA$ changes from 0.19 0.20 , i.e., is essentially model independent, while the iron abundance derived from Fe XX 101.55 $\AA$ varies from 0.06 to 0.24 in an extremely model dependent way.

If we now compare the abundances from the simplest acceptable discrete temperature component model (i.e., 4-T) to those derived from the simplest continuous emission measure distribution model $(4,40)$ we find that the nitrogen and oxygen abundances differ by a factor of $\approx 2$, the discrete temperature component abundances being lower. For the neon, magnesium, and silicon abundances the situation appears similar with the continuous temperature distribution abundances being higher, while for iron the two sets of abundances agree reasonably well, the main difference coming from the lines used for abundance analysis.

\subsection{Towards a physical model}

It should have become clear that neither the modeling approach based on Chebyshev polynomials nor that based on individual temperature components includes a great deal of physics other than the theory of hot thermal plasma emission. Let us consider a "standard" solar loop with apex temperature $T_{\text {max }}=2 \times 10^{6} \mathrm{~K}$, pressure $p=1 \mathrm{dyn} \mathrm{cm}^{-2}$, and loop half length $L=2 \times 10^{9} \mathrm{~cm}$. Such a structure contains a total emission measure of a few times $10^{46} \mathrm{~cm}^{-3}$. Comparing this estimate to the total solar coronal emission measure or to the emission measure observed from stars it is clear that several hundreds and possibly more than thousand such "standard" loops must be contributing to the emission observed at any given time. This is amply demonstrated by the thousands of $\mathrm{YOHKOH}$ and $\mathrm{SOHO}$ images, which show a vast variety of different emission structures, and only during a stronger flare an individual structure can dominate the total X-ray output. In a stellar context the situation is less clear. Again, during a large flare the overall emission is certainly dominated by the emission from the flare region alone, for the quiescent emission we assume that a larger number of individual features is responsible for the observed emission.

If one assumes that the X-ray emission comes from a sample of individual constant pressure "atmospheres" extending from some lower temperature $T_{0}$ to some maximum temperature $T_{\max }$, the differential emission measure distribution $\xi(T)$ of such a loop can be calculated as (cf. Bray 1991)

$$
\xi(T)=C_{\text {norm }} p T^{\alpha-\gamma / 2-1 / 4} \frac{1}{\sqrt{1-\left(\frac{T}{T_{\max }}\right)^{2-\gamma+\beta}}}
$$

The parameters $\alpha, \beta$, and $\gamma$ in Eq. (34) are the power law coefficients in the laws for radiative cooling, loop heating, and loop cross section expansion which are assumed to have the form

$\Lambda(T)=\Lambda_{0} T^{\gamma} \quad Q(T)=Q_{0} T^{\beta} \quad A(T)=A_{0} T^{\alpha}$,

respectively. Clearly, these assumptions are somewhat idealistic, and in particular the assumption about the heating law is totally arbitrary, but the resulting structure is found to be remarkably insensitive to the detailed form of these coefficients. The constant $C_{\text {norm }}$ in Eq. (34) is given by the expression

$C_{\mathrm{norm}}=A_{0} \sqrt{\frac{\kappa_{0}(2 \alpha+\gamma+3 / 2)}{8 \Lambda_{0} k^{2}}}$,

where $\kappa_{0}$ denotes the constant in the heat conduction law. The singularity at the loop top, where $T=T_{\max }$, arises from the boundary condition $\frac{\mathrm{d} T}{\mathrm{~d} s}=0$ at the same place. In such structures the fundamental physical parameters pressure, loop top temperature, and the loop length $L$ are not independent but are related through so-called scaling laws viz.

$p L \sim T_{\max }^{\frac{11-2 \gamma}{4}}$. 
In order to make progress we assume ad hoc that the distribution of the actual loop top temperatures $\Phi\left(T_{\max }\right)$ of a collection of loops can be described through a Gaussian viz.

$\Phi\left(T_{\max }\right)=\frac{1}{\sqrt{2 \pi} \sigma_{T}} \mathrm{e}^{-\frac{\left(T_{\max }-T_{0}\right)^{2}}{2 \sigma_{T}^{2}}}$,

with a mean loop top temperature $T_{0}$ and some dispersion $\sigma_{T}$. We further assume - for the time being - that all loops have the same length. It is clear that only loops with loop top temperature $T_{\max }>T$ can contribute to the overall differential emission measure at some given temperature $T$. The total differential emission measure from such a collection of loops is therefore given by

$$
\xi_{\text {tot }}(T) \sim \frac{1}{L} T^{\alpha-\gamma / 2-1 / 4} \int_{T}^{\infty} \mathrm{d} T_{\max } T_{\max }^{\frac{11-2 \gamma}{4}} \frac{\mathrm{e}^{-\frac{\left(T_{\max }-T_{0}\right)^{2}}{2 \sigma_{T}^{2}}}}{\sqrt{1-\left(\frac{T}{T_{\max }}\right)^{2-\gamma+\beta}}},
$$

where we have used the scaling law Eq. (37) to replace the (constant) loop pressure $p$ with $T_{\max }$ and $L$. Because of the scaling law Eq. (37) $p$ and $T_{\max }$ are not independent, and in fact for given $L$ the pressure and thus the total emission measure depend quite sensitively on $T_{\max }$. If we then - finally assume that the loop length $L$ is related to the loop top temperature with some power law coefficient $\delta$, we are led to the following analytical form of the differential emission measure distribution:

$\xi_{\text {tot }}(T)=A_{\text {norm }} T^{\alpha_{1}} \int_{T}^{\infty} \mathrm{d} T_{\max } \frac{T_{\max }^{\alpha_{2}}}{\sqrt{1-\left(\frac{T}{T_{\max }}\right)^{\alpha_{3}}}} \mathrm{e}^{-\frac{\left(T_{\max }-T_{0}\right)^{2}}{2 \sigma_{T}^{2}}}$,

with the three power law coefficients $\alpha_{1}, \alpha_{2}$, and $\alpha_{3}$, the mean loop top temperature $T_{0}$ and its variance $\sigma_{T}$ as parameters describing the shape of the distribution function.

With the Chandra LETGS data we can test whether the shape specified by Eq. (40) is consistent with our observations. Calculations showed that the hydrogen- to helium-like line ratios depend sensitively on $\alpha_{1}, T_{0}$, and $\sigma_{T}$, but less sensitively on the parameters $\alpha_{2}$ and $\alpha_{3}$. The best fit model was found to be given by the set $\alpha_{1}=0.48, \alpha_{2}=0.50, \alpha_{3}=0.50$, $T_{0}=7 \mathrm{MK}$, and $\sigma_{T}=11 \mathrm{MK}$. In Fig. 6 we plot the DEM distribution function in comparison to the best-fit DEM distributions reconstructed from fourth-order Chebyshev polynomials with $T_{\max }=30 \mathrm{MK}$ and $T_{\max }=40 \mathrm{MK}$; as is clear from Fig. 6, the DEM reconstructions agree extremely well with each other for temperatures above $1 \mathrm{MK}$, while the slope of the emission reconstructions disagrees for lower temperatures. Since there are almost no constraints on the DEM in this temperature range (note that the peak formation temperature for $\mathrm{N}$ VII is at $1 \mathrm{MK}$ ), such a discrepancy is hardly surprising. As a first conclusion we therefore note, that a function form as given in Eq. (40) provides a reasonable description of the observed line ratios with suitably chosen parameters $T_{0}, \sigma_{T}$, $\alpha_{1}, \alpha_{2}$, and $\alpha_{3}$. In the framework of the chosen model it is a little surprising that the mean (Gaussian) loop top temperature is rather low $(T=7 \mathrm{MK})$. However, because of the large dispersion of $11 \mathrm{MK}$, significantly hotter loops occur with high probability. Since according to the scaling laws higher loop

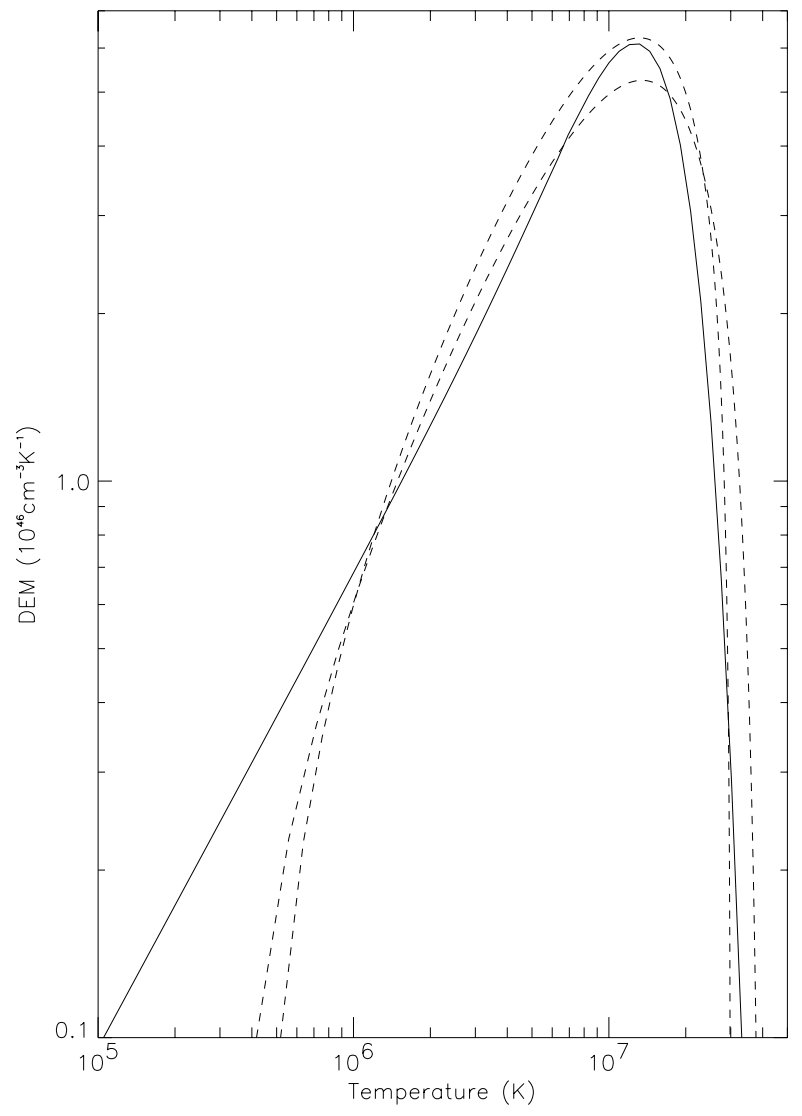

Fig. 6. Best fit differential emission measure distribution derived from Eq. (40) (solid curve) compared to DEM distributions derived from fourth order Chebyshev polynomials with $T_{\max }=30 \mathrm{MK}$ and $T_{\max }=$ $40 \mathrm{MK}$.

top temperatures imply higher pressure, higher pressure implies higher density, and higher density implies larger emission measure, so the hotter loop will contribute most. For constant cross section loops with the canonical radiative loss function one expects $\alpha_{1} \sim 0$; the observed value $\alpha_{1} \sim 0.5$ can only be explained by expanding loop geometries.

\section{Discussion and conclusions}

The new generation of X-ray spectrometers on board the Chandra and XMM-Newton satellites allows the determination of elemental abundances in hot X-ray emitting plasmas. The Chandra LETGS has the specific advantage of a very large band pass with an ensuing sensitivity to lines from rather different temperature regimes. Our analysis of the Algol LETGS spectrum shows that the abundances for the elements neon, magnesium, silicon, and iron are all sub-solar. This is in line with previously published high-resolution abundance determinations of HR1099 with the Chandra HETGS (Drake et al. 2001) and XMM-Newton (Brinkmann et al. 2001). Among those elements neon has the relatively highest abundance, i.e., it is least sub-cosmic. It appears that these conclusions are rather robust, and specifically do not sensitively depend on the methodology used ("global fit" vs. "emission line analysis"). However, an inspection of Table 2 shows that, for example, the iron abundance determinations considerably depend 
Table 4. Comparison of coronal abundances for Algol derived from Chandra LETGS (this paper, second column) with abundances derived from ASCA (third to fifth column; Antunes et al. 1994) and EUVE (sixth column; Stern et al. 1995).

\begin{tabular}{crcccc}
\hline \hline Element & This paper & $\begin{array}{c}\text { Antunes et al. } \\
\text { Low State }\end{array}$ & $\begin{array}{c}\text { Antunes et al. } \\
\text { Medium State }\end{array}$ & $\begin{array}{c}\text { Antunes et al. } \\
\text { High State }\end{array}$ & Stern et al. \\
\hline $\mathrm{C}$ & $<0.04$ & n.a. & n.a. & n.a. & n.a. \\
$\mathrm{N}$ & 2.0 & $<0.1$ & $<0.1$ & $<0.1$ & n.a. \\
$\mathrm{O}$ & 0.25 & $0.30 \pm 0.04$ & $0.31 \pm 0.03$ & $0.24 \pm 0.03$ & n.a. \\
$\mathrm{Ne}$ & 0.95 & $0.76 \pm 0.10$ & $1.22 \pm 0.08$ & $1.08 \pm 0.08$ & n.a. \\
$\mathrm{Mg}$ & 0.5 & $0.48 \pm 0.06$ & $0.64 \pm 0.05$ & $0.47 \pm 0.04$ & n.a. \\
$\mathrm{Si}$ & 0.45 & $0.43 \pm 0.05$ & $0.65 \pm 0.04$ & $0.47 \pm 0.03$ & n.a. \\
$\mathrm{Fe}$ & 0.2 & $0.30 \pm 0.01$ & $0.37 \pm 0.02$ & $0.32 \pm 0.01$ & $0.2-0.4$ \\
\hline
\end{tabular}

on the lines used for the analysis. The short wavelength lines of Fe XVII at 15.01 $\AA, 15.27 \AA$, and 17.07 $\AA$ yield higher abundances than the Fe lines at $93.92 \AA, 108.37 \AA$, $128.37 \AA$, and 132.82 $\AA$; the Fe line at 101.55 $\AA$ yields larger abundances than the rest of the EUV Fe lines. The reason(s) for this discrepancy are not quite clear. Optical depth effects in the Fe XVII are very likely not the cause (Ness et al. 2003) and would in fact even worsen the discrepancy. Since the short wavelength lines are all from Fe XVII, the calculated emission measure distribution for this ion might be incorrect, i.e., too large. Since Fe XVII is produced over a rather large temperature range this appears somewhat unlikely since then also the continuum emission would have to be incorrectly placed. The Fe XVII long wavelength lines are affected by absorption, but we used already a rather large value of $N_{\mathrm{H}}$; lowering the absorption column density would again worsen the discrepancy. Systematic errors in the instrument calibration might affect the long wavelength portion of the spectrum in a different way than the short wavelength portion, but the magnitude of the effect is much larger than the systematic calibration uncertainties $(\approx 15 \%)$. Finally, atomic physics uncertainties might affect the long wavelength lines different compared to the short wavelength lines. At any rate, we have to conclude and state that we presently have no satisfactory explanation for the discrepant abundance determinations for iron.

As to the abundance discrepancies derived from different analysis methods, our studies have clearly demonstrated the importance of the correct determination of the underlying temperature structure for a correct determination of elemental abundances. The cooling functions of individual lines contribute significantly over a temperature range of 0.3 dex and the shape of the emission measure distribution also implies considerable contributions far away from the peak formation temperatures of individual lines. The lines used in our study, i.e., $\mathrm{Ly}_{\alpha}$ and He-like $r$ lines, are formed over a rather wide temperature range, other lines, in particular lines from ions with incompletely filled shells, are formed over somewhat narrower temperature ranges necessitating an even better knowledge of the temperature structure. We purposely used only those lines for our differential emission measure reconstruction, because, first, these lines are among the strongest for each element and therefore the most likely lines to be detected in a recorded $\mathrm{X}$-ray spectrum, and second, the atomic physics of those lines ought to be known best. A reliable method for abundance determination must prevent any cross talk between the temperature and abundance structure of a plasma; therefore, the temperature structure should be determined independent from the elemental abundances either from line ratios of lines of the same chemical element (as done in this paper) or by using lines only from the same element (as done by, e.g., Drake et al. 2001). We next emphasize the need of physical considerations in the determination of the temperature structure. This is in particular required if one ever desires to determine elemental abundances in the Xray range with an accuracy achieved by optical abundance determinations. We have at the moment only few clues as to the differential emission measure distributions realized by stellar coronae and the uncertainties in our knowledge of the correct temperature structure prevents us from reaching precisely this goal. A modeling of the coronal emission in terms of individual temperature component is unsatisfactory from a physical point of view, from a procedural point of view and from a mathematical point of view. Abundances determined in this way may have small statistical errors (of a few percent depending on the SNR of the modeled data), but rather large systematic errors of $100 \%$ or more; nevertheless they are adequate to reveal general trends in abundance patterns. This is exemplified in Table 3, which shows that for example the oxygen abundance changes by a factor two for models with discrete temperature components. A comparison of the abundances derived for Algol in this paper with those derived by Antunes et al. (1994) from ASCA using a two-temperature variable abundance modeling approach also shows that the general trend in the run of elemental abundances is captured and the "low" iron abundance and "high" neon abundance are recognized, while the abundances of individual elements can vary by at least a factor of two. Also, the real clue of the Chandra LETGS Algol observation, i.e., the overabundance of nitrogen with its profound physical implications (cf. Schmitt \& Ness 2002) went unnoticed in the modeling with the lower resolution ASCA data.

As to Algol specifically, our detailed temperature and abundance modeling confirms the results previously derived by Schmitt \& Ness (2002). Because of temperature dependence of the emissivity functions of the $\mathrm{Ly}_{\alpha}$-lines for $\mathrm{C}$ and $\mathrm{N}$ (cf. Fig. 1), the line ratio between these lines must stay below 0.57 (for cosmic abundances) regardless of the underlying temperature structure in contrast to the observed ratio of $>23$. Our modeling now shows that carbon is depleted down to at least $8 \%$, while nitrogen is enhanced by about $70 \%$ or more (all relative to cosmic abundances). This effect is dramatic. Assuming the cosmic abundance pattern recommended by Holweger (2001) 
there are 4.58 carbon atoms for every nitrogen atom, while in Algol's corona we have (at least) 8.2 nitrogen atoms for every carbon atom! This reversal of carbon and nitrogen abundance can be readily explained by assuming that one is studying CNO-cycle processed material in the corona of Algol B, since the equilibrium abundance of $\mathrm{CNO}$ nuclei participating in the cycle is such that most nuclei occur as $\mathrm{N}^{14}$ nuclei (Caughlan 1965). In no other spectral range than the X-ray band can the chemical abundance of the B component of the Algol system be studied.

Our Chandra LETGS spectrum of Algol thus demonstrates the wealth of physical information contained in an X-ray spectrum with high spectral resolution and - at the same time good signal-to-noise ratio. The latter is as important as the former, since data with poor signal-to-noise will not allow the derivation of meaningful and significant results. The exposure of such spectra requires substantial satellite resources, yet it represents the only way to extract information on the physics of stellar coronae.

Acknowledgements. J.-U. Ness acknowledges suppert from the DLR grant 50OR0105. We acknowledge useful discussions and help from Drs. P. Predehl and V. Burwitz.

\section{References}

Allen, C. W. 1973, in Astrophysical Quantities (University of London: The Athlone Press)

Antunes, A., Nagase, F., \& White, N. E. 1994, ApJ, 436, L83

Audard, M., Güdel, M., \& Mewe, R. 2001, A\&A, 365, L318

Arnaud, M., \& Rothenflug, R. 1985, A\&AS, 60, 425

Bray, R. J., Cram, L. E., Currant, C. J., \& Loughhead, R. E. 1991, Plasma Loops in the Solar Corona (Cambridge University Press, ISBN 052135197)

Brinkman, A. C., Behar, E., Güdel, M., et al. 2001, A\&A, 365, L324

Caughlan, G. R. 1965, ApJ, 141, 688

Craig, I. J. D., \& Brown, J. C. 1976, Nature, 264, 340

Dere, K. P., Landi, E., Mason, H. E., Monsignori Fossi, B. C., \& Young, P. R. 1997, A\&AS, 125, 149
Drake, J. J., Laming, J. M., \& Widing, K. G. 1996, in Astrophysics in the Extreme Ultraviolet, IAU Colloq., 152, 97

Drake, J. J., Brickhouse, N. S., Kashyap, V., et al. 2001, ApJ, L81

Drake, J. J. 2003, ApJ, 594, 496

Grevesse, N., \& Sauval, A. J. 1998, Space. Sci. Rev., 85, 161

Güdel, M., Audard, M., Briggs, K., et al. 2001, A\&A, 365, L336

Güdel, M., Audard, M., Magee, H., et al. 2001, A\&A, 365, L344

Huenemoerder, D. P., Canizares, C. R., \& Schulz, N. S. 2002, ApJ, 559,1135

Favata, F., \& Schmitt, J. H. M. M. 1999, A\&A, 350, 900

Fludra, A., \& Schmelz, J. T. 1995, ApJ, 447, 936

Huenemoerder, D. P., Canizares, C. C., \& Schulz, N. S. 2001, ApJ, 559,1135

Holweger, H. 2001, Joint SOHO/ACE workshop Solar and Galactic Composition, ed. F. Robert, Wimmer-Schweingruber, American Institute of Physics, Conf. Proc. 598, Bern, Switzerland, March 69, 2001, 23

Lemen, J. R., Mewe, R., Schrijver, C. J., \& Fludra, A. 1989, ApJ, 341, $474 \mathrm{~L}$

Mazzotta, P., Mazzitelli, G., Colafrancesco, S., \& Vittorio, N. 1998, A\&AS, 133, 403

McIntosh, S. W. 2000, ApJ, 533, 1043

Mewe, R., Gronenschild, E. H. B. M., \& van den Oord, G. H. J. 1985, A\&AS, 62, 197

Mewe, R., Lemen, J. R., \& van den Oord, G. H. J. 1986, A\&AS, 65, 511

Ness, J.-U., Schmitt, J. H. M. M., Burwitz, V., et al. 2002a, A\&A, 387, 1032

Ness, J.-U., Schmitt, J. H. M. M., Burwitz, V., et al. 2002b, A\&A, 394, 911

Ness, J.-U., Schmitt, J. H. M. M., Audard, M., \& Güdel, M. 2003, A\&A, submitted

Ottmann, R., \& Schmitt, J. H. M. M. 1996, A\&A, 307, 813

Pottasch, S. R. 1965, IAU Symp., 23, ed. J.-L. Steinberg., 105

Raymond, J. C., \& Smith, B. W. 1977, ApJS, 35, 419

Schmitt, J. H. M. M., Stern, R. A., Drake, J. J., \& Kürster, M. 1996, ApJ, 464, 898

Schmitt, J. H. M. M., \& Ness, J.-U. 2002, A\&A, 388, L13

Stern, R. A., Lemen, J. R., Schmitt, J. H. M. M., \& Pye, J. P. 1995, ApJ, 444, L45

White, N. E., Arnaud, K., Day, C. S. R., et al. 1994, PASJ, 46, L97

Young, P. R., Del Zanna, G., Landi, E., et al. 2003, ApJS, 144, 135 